\title{
Visceral Leishmaniasis Associated with Hemophagocytic Lymphohistiocytosis
}

\author{
Rishi Bolia ${ }^{1}$ (I) $\cdot$ Avinish Singh ${ }^{1} \cdot$ Manideepa Maji $^{1} \cdot$ Medha Misra $^{2} \cdot$ Indar Kumar Sharawat ${ }^{1} \cdot$ Prateek Kumar Panda $^{1}$
}

Received: 22 March 2020 / Accepted: 19 May 2020 / Published online: 3 June 2020

(C) Dr. K C Chaudhuri Foundation 2020

To the Editor: An 18-mo-old toddler hailing from Nepal presented to us with persistent fever of 4 mo duration, anemia and splenohepatomegaly. Laboratory studies demonstrated pancytopenia (hemoglobin $8.2 \mathrm{~g} / \mathrm{dl}$, TLC 3000/ $\mathrm{mm}^{3}$, platelets $72,000 / \mathrm{mm}^{3}$ ), hypertriglyceridemia $(742 \mathrm{mg} / \mathrm{dl})$, hyperferritinemia $(2075 \mathrm{ng} / \mathrm{ml})$ with features of hemophagocytosis on bone marrow aspiration (BMA) suggesting a diagnosis of hemophagocytic lymphohistiocytosis (HLH), as it met $\geq 5$ out of 8 of the HLH diagnostic criteria. Investigations for malaria, Epstein-Barr virus and HIV were negative. The Anti-rK39 ELISA was positive. On this basis, a diagnosis of Visceral leishmaniasis (VL) with secondary HLH was made. The patient was treated with single dose $10 \mathrm{mg} / \mathrm{kg}$ liposomal amphotericin B (LamB). Child showed clinical improvement and on follow-up after $8 \mathrm{wk}$ there was no fever, spleen size had significantly decreased and blood counts had normalized.

$\mathrm{VL}$ is caused by the protozoa Leishmania donovani (LD) and is an uncommon cause of HLH, representing $2.1 \%$ of all such cases [1]. VL-HLH poses as diagnostic dilemma as features of VL and HLH overlap, often delaying the diagnosis.

Only a handful of cases of pediatric VL-HLH have been reported in literature with the median age of diagnosis being $2 \mathrm{y}$ [2]. However, it might be under-recognized rather than being uncommon as a prospective multicentre study demonstrated that up to $41 \%$ of children with VL developed HLH [3].

In our child, BMA did not demonstrate LD bodies which are diagnostic of VL. They may not be seen in up to $36 \%$ cases of VL because of the paucimicrobial nature of the disease. The

Rishi Bolia

rishibolia@yahoo.co.in

1 Department of Pediatrics, All India Institute of Medical Sciences, Rishikesh, India

2 Department of Pathology, All India Institute of Medical Sciences, Rishikesh, India
Anti-rK39 ELISA has 97\% sensitivity and 98.9\% specificity and is a valuable tool in diagnosing VL [4].

Clavijo et al. studied children with VL, with and without HLH and found that there were no differences in outcome [1]. Specific treatment for HLH is generally not needed and most respond to treatment for VL alone as seen in our patient. We used only a single dose of LamB which we believe is sufficient, though others have used longer courses $[2,5]$.

This case illustrates the importance of recognizing VL as an etiology of $\mathrm{HLH}$, especially in patients from endemic areas and timely administering single-dose LamB to prevent lifethreatening complications.

\section{Compliance with Ethical Standards}

Conflict of Interest None.

\section{References}

1. Clavijo A, Salvador T, Moral L, Gavilan C, Squittieri C, Ramos JM. Hemophagocytic lymphohistiocytosis in children with visceral leishmaniasis. Pediatr Infect Dis J. 2016;35:713-4.

2. Scalzone M, Ruggiero A, Mastrangelo S, et al. Hemophagocytic lymphohistiocytosis and visceral leishmaniasis in children: case report and systematic review of literature. J Infect Dev Ctries. 2016;10: 103-8.

3. Blázquez-Gamero D, Domínguez-Pinilla N, Chicharro C, et al; Madrid Leishmaniasis Study Group. Hemophagocytic lymphohistiocytosis in children with visceral leishmaniasis. Pediatr Infect Dis J. 2015;34:667-9.

4. Rajagopala S, Dutta U, Chandra KS, Bhatia P, Varma N, Kochhar R. Visceral leishmaniasis associated hemophagocytic lymphohistiocytosis-case report and systematic review. J Inf Secur. 2008;56:381-8.

5. Pandey K, Pal B, Siddiqui NA, et al. Efficacy and safety of liposomal amphotericin B for visceral leishmaniasis in children and adolescents at a tertiary care center in Bihar, India. Am J Trop Med Hyg. 2017;97:1498-502.

Publisher's Note Springer Nature remains neutral with regard to jurisdictional claims in published maps and institutional affiliations. 